\title{
RBFOX2 and alternative splicing in B-cell lymphoma
}

\author{
Hilmar Quentmeier ${ }^{1}$, Claudia Pommerenke', Stephan H. Bernhart ${ }^{2}$, Wilhelm G. Dirks', Vivien Hauer', Steve Hoffmann ${ }^{3}$, \\ Stefan Nagel ${ }^{1}$, Reiner Siebert ${ }^{4}$, Cord C. Uphoff ${ }^{1}$, Margarete Zaborski ${ }^{1}$ and Hans G. Drexler ${ }^{1}$, ICGC MMML-Seq \\ Consortium
}

RBFOX2 is a master regulator of alternative splicing ${ }^{1}$. This RNA-binding protein (RBP) is expressed in the brain $^{2}$, muscle ${ }^{3}$, and embryonic stem cells ${ }^{4}$. RBFOX2 is required not only for the proper splicing of target RNAs, but also for cerebellar development ${ }^{2}$, myogenesis ${ }^{3}$, and for survival of human embryonic stem cells ${ }^{4}$.

Hitherto, not much is known about the expression and function of RBFOX2 in hematopoetic tissues. In an early report, RBFOX2 had been shown to be capable of promoting inclusion of exon 16 in protein $4.1 \mathrm{R}^{5}$. This splicing event is important for erythropoiesis because it increases the affinity of $4.1 \mathrm{R}$ for target genes ${ }^{5}$. In a recent study, expression of RBFOX2 was detected in the human T-cell line JURKAT and a functional antagonism of the RBPs RBFOX2 and CELF2 was demonstrated ${ }^{6}$. We wanted to describe the expression patterns of RBFOX2 in hematopoetic malignancies, to discover target genes and to unravel the consequence of $R B F O X 2$ repression for target gene splicing and isoform expression.

Expression array and Western blot analysis showed that human B non-Hodgkin lymphoma (B-NHL) cell lines are RBFOX2 negative or positive (Supplement $1 \mathrm{~A}$ ). To find the potential targets of the splice factor $R B F O X 2$, we compared the expression of individual exons in RBFOX2negative and RBFOX2-positive cell lines. This approach relied on the assumption that the differential expression of this RBP would provoke changes in the expression of individual exons and would thereby allow identification of target genes. Supplement 1B shortlists these genes

\footnotetext{
Correspondence: Hilmar Quentmeier (hqu@dsmz.de)

'Department of Human and Animal Cell Lines, Leibniz-Institute DSMZ-German Collection of Microorganisms and Cell Cultures, Braunschweig, Germany

${ }^{2}$ Transcriptome Bioinformatics Group - Interdisciplinary Centre for Bioinformatics, Leipzig University, Leipzig, Germany

Full list of author information is available at the end of the article.
}

ordered by statistical significance. Expression of the individual exons and joining sequences of MALT1 is shown as heatmap in Fig. 1a. The full-length MALT1 variant was associated with RBFOX2 expression (Fig. 1a).

Results of splice variant analysis with a larger panel of cell lines revealed a striking association between expression of $R B F O X 2$ and expression of the full-length forms of all four candidate target genes, MALT1, CLSTN1, FMNL3, and $M Y O 9 B$ (Fig. 1b). The short variants were expressed in RBFOX2-negative cell lines only (Fig. 1b). Two of these potential RBFOX2 target genes (CLSTN1 and FMNL3) had already been described in the context of RBFOX2mediated splicing ${ }^{7}$. The RBFOX2 target sequence "UGCAUG" was present in all introns following the retained exons, indicating that high $R B F O X 2$ levels might be the cause of the full-length forms in the RBFOX2 positive cell lines. Supporting the notion that RBFOX2 was important for splicing of these genes was also the finding that RBFOX2 was the sole gene that was significantly overexpressed in cell lines expressing full-length MALT1 when compared to cell lines expressing MALT1 without exon 7 (Supplement 1C).

We performed knockdown experiments to test whether RBFOX2 was responsible for retaining MALT1 exon 7, $F M N L 3$ exon 26, and $M Y O 9 B$ exon 37. siRNAs reduced expression of RBFOX2 in RBFOX2-positive cell lines BL2, SU-DHL-5, and HT by more than 50\% (Fig. 1c, Supplement 1D). Repression of RBFOX2 induced the shorter isoforms of MALT1 (w/o exon 7), MYO9B (w/o exon 37), and FMNL3 (w/o exon 26) (Fig. 1c, Supplement 1D). The long form of CLSTN1, the fourth gene tested here, was not or only marginally expressed in BL-2, SU-DHL-5, and HT cells, explaining why we could not observe an increase of the short isoform of this gene after RBFOX2 knockdown (data not shown). In sum, our data showed that 


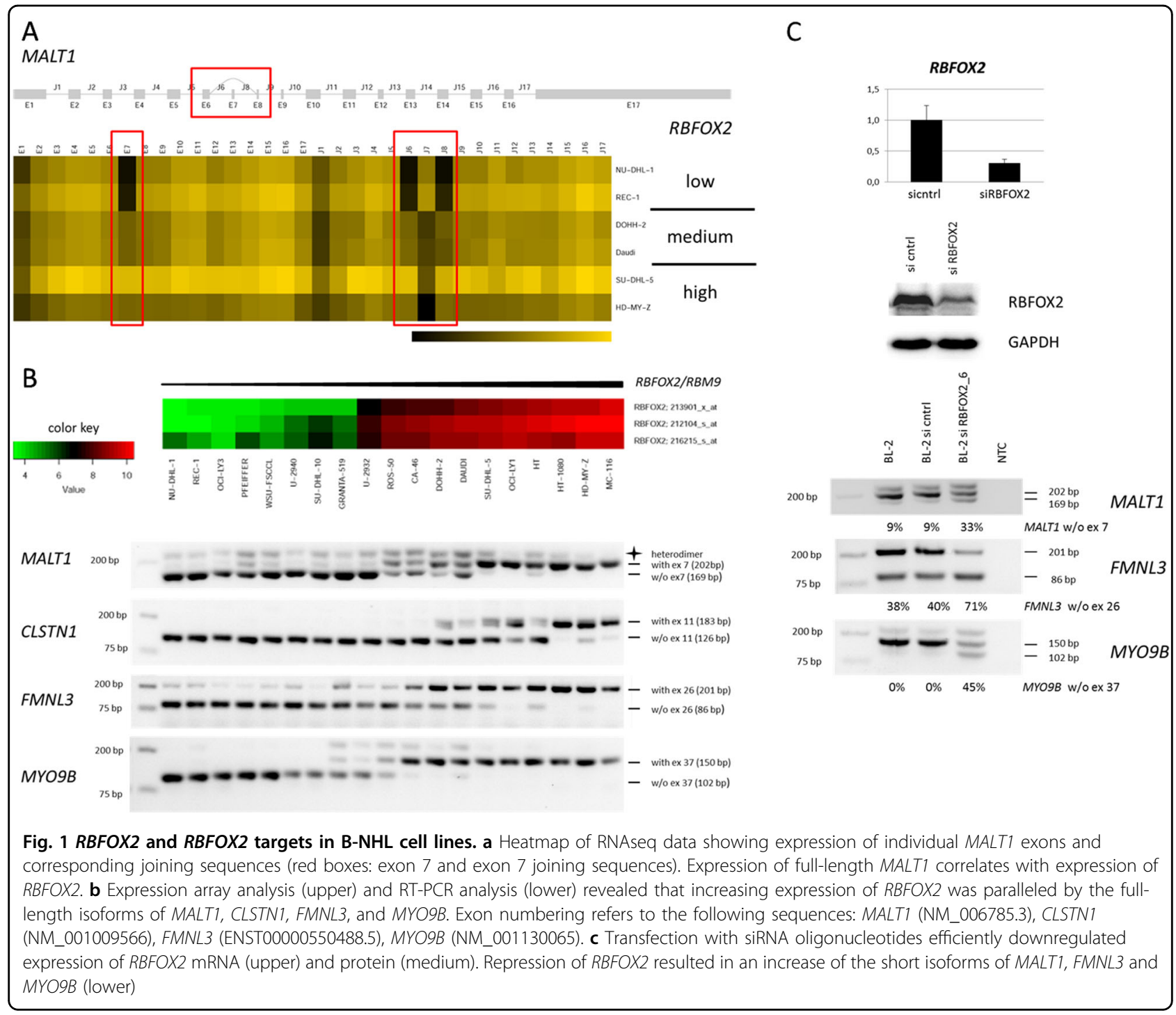

isoforms of MALT1, MYO9B, and FMNL3 in B-NHL cell lines are controlled by RBFOX2: the full-length RNAs were expressed when $R B F O X 2$ was high, the short variants prevailed when $R B F O X 2$ was repressed.

RBFOX2 is part of a mesenchymal splicing network ${ }^{7}$. The gene is also essential for the viability of human embryonic stem cells and for normal cerebellar development of mice ${ }^{2,4}$. Thus, RBFOX2 appears to have different functions in cells of different origin. To check whether RBFOX2 induced splicing of MALT1, CLSTN1, FMNL3, and $M Y O 9 B$ in hematopoetic cells other than B-cells, we tested RBFOX2 expression and expression of the splice variants of the putative RBFOX2 targets in T- and myeloid cell lines.

Neither T-cell lines nor myeloid cell lines reached the RBFOX2 mRNA expression level of the B-cell line HT. Nevertheless, the T- and myeloid cell lines with highest RBFOX2 mRNA levels also expressed the protein
(Supplement 1A). T-cell lines with high RBFOX2 levels expressed the large isoform of MALT1. However, an apparent universal dependence of $R B F O X 2$ and the large isoforms of MALT1, CLSTN1, FMNL3, and MYO9B comparable to that in B-cell lines was neither found in $\mathrm{T}$ nor in myeloid cell lines. This does not necessarily mean that RBFOX2 is functionless in cells of the T-lymphoid and myeloid lineages. RBFOX2 is expressed in cell lines of both entities, and the full-length MALT1 isoform is expressed in RBFOX2-positive T-cells. The other three genes (CLSTN1, FMNL3, and MYO9B) appear to be targets of RBFOX2 in the $\mathrm{B}$-lineage only. These results suggest that tissue-specific factors might contribute to the splicing process mediated by RBFOX2.

We limited our further studies to B-NHL, because we had identified the RBFOX2 target genes in B-NHL cell lines. As shown in cell lines, also primary tumor cells of patients with diffuse large B-cell lymphoma (DLBCL) 
Table 1 Correlation between expression of RBFOX2 and inclusion of exons in RBFOX2 target genes

\begin{tabular}{|c|c|c|c|c|c|c|c|c|}
\hline & \multicolumn{2}{|c|}{ MALT1 exon 7} & \multicolumn{2}{|c|}{ CLSTN1 exon 11} & \multicolumn{2}{|c|}{ FMNL3 exon 26} & \multicolumn{2}{|c|}{ MYO9B exon 37} \\
\hline & Correlation & $p$-value & Correlation & $p$-value & Correlation & $p$-value & Correlation & $p$-value \\
\hline DLBCL ABC $(n=26)$ & 0.496 & 0.009 & 0.450 & 0.023 & 0.139 & 0.496 & 0.338 & 0.09 \\
\hline DLBCL GCB $(n=37)$ & 0.591 & 0.0001 & 0.147 & 0.383 & 0.558 & 0.0004 & 0.561 & 0.0003 \\
\hline DLBCL type III $(n=15)$ & 0.125 & 0.675 & 0.385 & 0.156 & 1 & 0 & 0.307 & 0.265 \\
\hline All DLBCL $(n=78)$ & 0.522 & $1.2 \times 10^{-6}$ & 0.303 & 0.007 & 0.300 & 0.008 & 0.566 & $1.1 \times 10^{-7}$ \\
\hline $\mathrm{BL}$ (solid ped BL) $(n=21)$ & 0.703 & 0.0005 & 0.669 & 0.001 & 0.484 & 0.027 & 0.672 & 0.001 \\
\hline $\mathrm{FL}(n=87)$ & 0.321 & 0.003 & 0.437 & $2.3 \times 10^{-5}$ & 0.227 & 0.034 & 0.397 & 0.0001 \\
\hline FL-DLBCL $(n=15)$ & 0.464 & 0.083 & 0.029 & 0.923 & 0.503 & 0.058 & 0.435 & 0.106 \\
\hline GC B cells, control $(n=5)$ & -0.9 & 0.083 & 0.2 & 0.783 & 0.3 & 0.683 & -0.2 & 0.783 \\
\hline Naive B cells, control $(n=5)$ & 0.6 & 0.083 & -0.354 & 0.783 & -0.8 & 0.683 & -0.2 & 0.783 \\
\hline
\end{tabular}

RNASeq data from lymphoma and control, mapped to hg38 with segemehl 2.0; data were normalized against target gene expression. Bold: statistically significant. Normalization: transformation to target gene expression levels

show differential RBFOX2 gene expression (Fig. 1b upper, Supplement 1E). We analyzed RNAseq data from patients with different forms of B-NHL (ICGC MMML-Seq consortium) to find out whether primary tumor cells exhibited the same correlation between RBFOX2 expression and the RBFOX2 target gene isoforms as detected in $\mathrm{B}-$ NHL cell lines. We checked samples from patients with DLBCL $(n=78)$, Burkitt lymphoma (BL) $(n=21)$, follicular lymphoma (FL) $(n=87)$, and FL-DLBCL $(n=15)$. Germinal center (GC) B-cells $(n=5)$ and naive B-cells $(n=5)$ were included as controls.

RBFOX2 expression and MALT1 exon 7 inclusion were positively correlated in $\mathrm{BL}, \mathrm{FL}$, activated $\mathrm{B}$-cell $(\mathrm{ABC})$, and GC DLBCL $(p<0.05)$ (Table 1). In contrast, no such correlation was found for healthy controls, DLBCL (type III) and FL-DLBCL (Table 1). Supporting the notion that RBFOX2 regulates splicing in all four proposed $R B F O X 2$ target genes (MALT1 exon 7, CLSTN1 exon 11, FMNL3 exon 26, and $M Y O 9 B$ exon 37), we found a statistically significant positive correlation between expression of RBFOX2 and inclusion of target exons in $\mathrm{FL}, \mathrm{BL}$, and in at least one subtype of DLBCL (Table 1). The data had been normalized against target gene expression levels to avoid a potential bias through target gene expression levels.

$R B F O X 2$ is a member of the RBFOX family of RBP, also including $R B F O X 1$ and $R B F O X 3$. All three proteins recognize the same sequence (UGCAUG) in regulated exons or in flanking introns ${ }^{8}$. To analyze whether RBFOX1 and RBFOX 3 might also contribute to the splicing of our four target genes, we tested for correlation between expression of these RBFOX family members and inclusion of exons in target genes. We did not find a statistically significant correlation between RBFOX1 or RBFOX3 expression and inclusion of exons in CLSTN1 and FMNL3 (Supplement 1F). MALT1 and MYO9B showed this correlation only in selected tumor variants, but not in BL, ABC DLBCL, or GC DLBCL, when the latter two were analyzed as individual lymphoma entities (Supplement 1F). Thus, RBFOX2 was the only RBFOX family member whose expression was positively correlated with the full-length isoforms of the target genes (MALT1, CLSTN1, FMNL3, and MYO9B) in BL, FL, and DLBCL.

These data suggest that RBFOX2 is a regulator of splicing in B-NHL. This notion is based on (i) the positive correlation between RBFOX2 expression and expression of the full-length variants of the putative RBFOX2 target genes in B-NHL cell lines and in primary B-NHL samples, and (ii) results of knockdown experiments demonstrating that RBFOX2 is responsible for inclusion of exons in $M A L T 1$ and other target genes.

MALT1 appears to be of special interest as it encodes a protease that activates the IKK complex ${ }^{9}$. In lymphocytes, MALT1 cleaves RelB, which also leads to the activation of $\mathrm{NFkB}^{10}$. Both MALT1 isoforms (with and w/o exon 7) are expressed in T-lymphocytes and expression of the individual variants has consequences for $\mathrm{T}$-cell receptor triggered signal transduction ${ }^{11}$. As part of the CARMA1-BCL10-MALT1 complex, MALT1 is also a central regulator of the B-cell receptor (BCR) / NFkB pathway. ABC-type DLBCL cells rely on the constitutive activation of this pathway to block apoptosis ${ }^{12}$. Recurrent mutations in $C D 79 A / B, C A R D 11$, and other BCR/NFkB pathway genes have been described ${ }^{13}$. Like Brutons Tyrosine Kinase, upstream to MALT1 in the BCR/NFkB pathway, also MALT1 is a potential target for precision therapy ${ }^{14}$. Future studies shall elucidate whether the two MALT1 isoforms display different capacities to activate NFkB in B-NHL, which might be of importance for the clinical application of MALT1 inhibitors. 
In summary, (i) RBFOX2 is expressed in hematopoetic cell lines of different origin; (ii) expression of RBFOX2 correlates with isoforms of potential target genes in BNHL cell lines and in primary B-NHL cells; and (iii) knockdown experiments suggest that RBFOX2-directly or indirectly-contributes to the splicing of target genes including $M A L T 1$, a protease in the $\mathrm{BCR} / \mathrm{NFkB}$ pathway.

\section{Acknowledgements}

The authors gratefully acknowledge the Gauss Centre for Supercomputing e.V. (www.gauss-centre.eu) for funding this project by providing computing time on the GCS Supercomputer SuperMUC at Leibniz Supercomputing Centre (www.lrz.de). This study has been supported by the German Ministry of Science and Education (BMBF) in the framework of the ICGC MMML-Seq project (01KU1002A-J), the project ICGC DE-MINING (01KU1505G), and the KinderKrebsInitiative Buchholz/Holm-Seppensen.

\section{Author details}

'Department of Human and Animal Cell Lines, Leibniz-Institute DSMZ-German Collection of Microorganisms and Cell Cultures, Braunschweig, Germany.

${ }^{2}$ Transcriptome Bioinformatics Group - Interdisciplinary Centre for Bioinformatics, Leipzig University, Leipzig, Germany. ${ }^{3}$ Computational Biology, Leibniz Institute on Aging - Fritz Lipmann Institute and Friedrich Schiller University Jena, Jena, Germany. ${ }^{4}$ Institute of Human Genetics, UIm University and UIm University Medical Center, UIm, Germany

\section{Author contributions}

H.Q. Study conception and design, manuscript writing. C.P.: Analysis of RNAseq data, expression array analysis, statistical analysis. S.H.B.: Analysis of RNAseq data, statistical analysis. W.G.D.: Knockdown experiments. V.H.: Acquisition of data. S.H.: RNAseq data analysis. S.N.: Knockdown experiments. R.S.: Coordination of the ICGC-MMML-Seq and the primary lymphoma data generation and provision, interpretation of data. C.C.U.: Analysis and presentation of data. M.Z: Acquisition of data. H.G.D.: Provision of cell lines and study conception. All authors read and approved the final manuscript.

\section{Conflict of interest}

The authors declare that they have no conflict of interest.

\section{Publisher's note}

Springer Nature remains neutral with regard to jurisdictional claims in published maps and institutional affiliations.
Supplementary Information accompanies this paper at (https://doi.org/ 10.1038/s41408-018-0114-3).

Received: 27 February 2018 Revised: 20 June 2018 Accepted: 6 July 2018 Published online: 10 August 2018

\section{References}

1. Arya, A. D., Wilson, D. I., Baralle, D. \& Raponi, M. RBFOX2 protein domains and cellular activities. Biochem. Soc. Trans. 42, 1180-1183 (2014).

2. Gehman, L. T. et al. The splicing regulator Rbfox2 is required for both cerebellar development and mature motor function. Genes Dev. 26, 445-460 (2012).

3. Singh, R. K. et al. Rbfox2-coordinated alternative splicing of Mef2d and Rock2 controls myoblast fusion during myogenesis. Mol. Cell 55, 592-603 (2014).

4. Yeo, G. W. et al. An RNA code for the FOX2 splicing regulator revealed by mapping RNA-protein interactions in stem cells. Nat. Struct. Mol. Biol. 16, 130-137 (2009).

5. Ponthier, J. L. et al. Fox-2 splicing factor binds to a conserved intron motif to promote inclusion of protein 4.1R alternative exon 16. J. Biol. Chem. 281, 12468-12474 (2006).

6. Gazzara, M. R. et al. Ancient antagonism between CELF and RBFOX families tunes mRNA splicing outcomes. Genome Res. 27, 1360-1370 (2017).

7. Venables, J. P. et al. MBNL1 and RBFOX2 coopertate to establish a splicing programme involved in pluripotent stem cell differentiation. Nat. Commun. 4, 2480 (2013).

8. Kuroyanagi, H. Fox-1 family of RNA-binding proteins. Cell. Mol. Life Sci. 66, 3895-3907 (2009).

9. McAllister-Lucas, L. M. \& Lucas, P. C. Finally, MALT1 is a protease. Nat. Immunol. 9. 231-233 (2008).

10. Hailfinger, S. et al. MALT1-dependent RelB cleavage promotes canonical NF-kB activation in lymphocytes and lymphoma cell lines. Proc. Natl. Acad. Sci. USA. 108, 14596-14601 (2011).

11. Meininger, I. et al. Alternative splicing of MALT1 controls signalling and activation of CD4+T cells. Nat. Commun. 7, 11292 (2016).

12. Davis, R. E., Brown, K. D., Siebenlist, U. \& Staudt, L. M. Constitutive nuclear factor $\mathrm{kB}$ activity is required for survival of activated B cell-like diffuse large B cell lymphoma cells. J. Exp. Med. 194, 1861-1874 (2001).

13. Pasqualucci, L. \& Dalla-Favera, R. SnapShot: Diffuse large B cell lymphoma. Cancer Cell. 25, 132-132e1 (2014).

14. Nagel, D. et al. Pharmacologic inhibition of MALT1 protease by phenothiazines as a therapeutic approach for the treatment of aggressive ABC-DLBCL. Cancer Cell. 22, 825-837 (2012) 